\title{
Small-Bowel Myeloid Sarcoma Presenting as A Relapse Case of Acute Myeloid Leukemia
}

\author{
Nadia Shirazi*, Akriti Saxena, Kunal Das and Vikas Shrivastava \\ Department of Pathology, Himalayan Institute of Medical Sciences, Swami Rama Himalayan University, \\ Jolly Grant, Dehradun. Uttarakhand. India.
}

\begin{abstract}
Granulocytic sarcoma is an extramedullary tumor of primitive granulocytic cells. It can develop at any anatomic site and is often a prelude to the development of Acute MyelogenousLeukaemia. Granulocytic sarcoma of the small intestine presents with abdominal pain and obstruction. We report a case of a 17 year old boy who presented with epigastric pain. His endoscopy revealed nodular duodenal mucosa and an area of ulcerated exophytic growthin the small bowel. Histopathology and flowcytometery confirmed the diagnosis of granulocytic sarcoma associated with acute myelogenousleukaemia. This case merits interest because of rarity of presentation and thorough clinical and laboratory work up.
\end{abstract}

Keywords: Extramedullary, Myeloblasts, Acute Leukemia, Flow Cytometry

\section{Introduction}

Granulocytic sarcoma/myeloid sarcoma (GS) is an uncommon tumor composed of myeloid blasts and/or immature myeloid cells at an extramedullary site. ${ }^{[1]} \mathrm{GS}$ may present in association with acute myeloid leukemia (AML), either as an initial presentation or as a relapse. Previous terms used to describe this entity include extramedullary myeloid tumor, granulocytic sarcoma (GS), and chloroma. The word chloroma was coined initially as these neoplasms had a gross greenish appearance due to large amount of Myeloperoxidase present in these cells. It was first described by Burns in 1811. ${ }^{[1]}$ Davey in 1988 has proposed the term extramedullary myeloid tumour (EMMT) to include all forms of extramedullary myeloid leukaemic infiltrates. ${ }^{[2]}$ The incidence rate of GS is about $2-5 \%$ in adults . In children it is significantly higher as it reaches $40 \%$ in patients with AML.It can occur at any anatomical site, most common sites being the skin $(13 \%$ $22 \%$ ), bone/spine $(9 \%-25 \%)$, and lymph nodes $(15 \%-$ $25 \%$ ). Involvement of gastrointestinal tract is rare $(7 \%)$ with the commonest site being ileum (10\%-11\%) followed by stomach and large intestine.$^{[3]}$ Clinical manifestation is often vague abdominal pain with or without obstruction, very rarely it may present with perforation and bleeding. We are reporting herewith a partly treated case of AML who presented with small bowel obstruction and perforation.

\section{Case Report}

A 17 year old male who was diagnosed AML on bone marrow examination an year backpresented with high grade fever,colicky pain, abdominal tenderness and vomiting since 4 days. Patient had taken 3 cycles of chemotherapy His Total Leukocyte Count (TLC) at the time of presentation was $>4$ lacs with $90 \%$ blasts. Contrast Enhanced Computerised Tomography (CECT) abdomen showed hepatosplenomegaly, inguinal \& retroperitoneal lymphadenopathy, large collection of fecal matter in retroperitoneum with extensions in left iliac fossa and descending colon along with mild ascites (Figure 1).

Exploratory laparotomy was performed which revealed an area of perforation in ileum. Dense inter bowel adhesions were seen along with collection of pus and fecal matter in the retroperitoneum. Resection anastomosis of intestinal segment was done and specimen was sent for histopathology.Grossly a segment of ileum was received measuring $6 \times 4 \times 2 \mathrm{~cm}$. Outer surface showed an area of perforation measuring $3.5 \times 2 \mathrm{~cm}$. The perforation was $1 \mathrm{~cm}$ and $4 \mathrm{~cm}$ from the random resected margins. Rest of the mucosa was edematous and congested. (Figure 2) Microscopically it showed a large area of mucosal ulceration covered with fibrinous exudate. Just beneath the ulcer loosely cohesive large atypical cells were seen having high Nucleo-cytoplasmic (N:C) ratio, convoluted nuclei and prominent nucleoli. (Figure 3). These cells were Myeloperoxidase (MPO) positive. Diagnosis of granulocytic sarcoma was rendered on histopathology. Flow cytometry was done which showed MPO, CD13 and CD33 positivity thus confirming a diagnosis of AML M1. (Figure 4)

\section{Discussion}

Myeloid sarcoma (MS) can occur concomitantly or can precede $\mathrm{AML}$ in $2 \%-7 \%$. It can even present as a relapse 


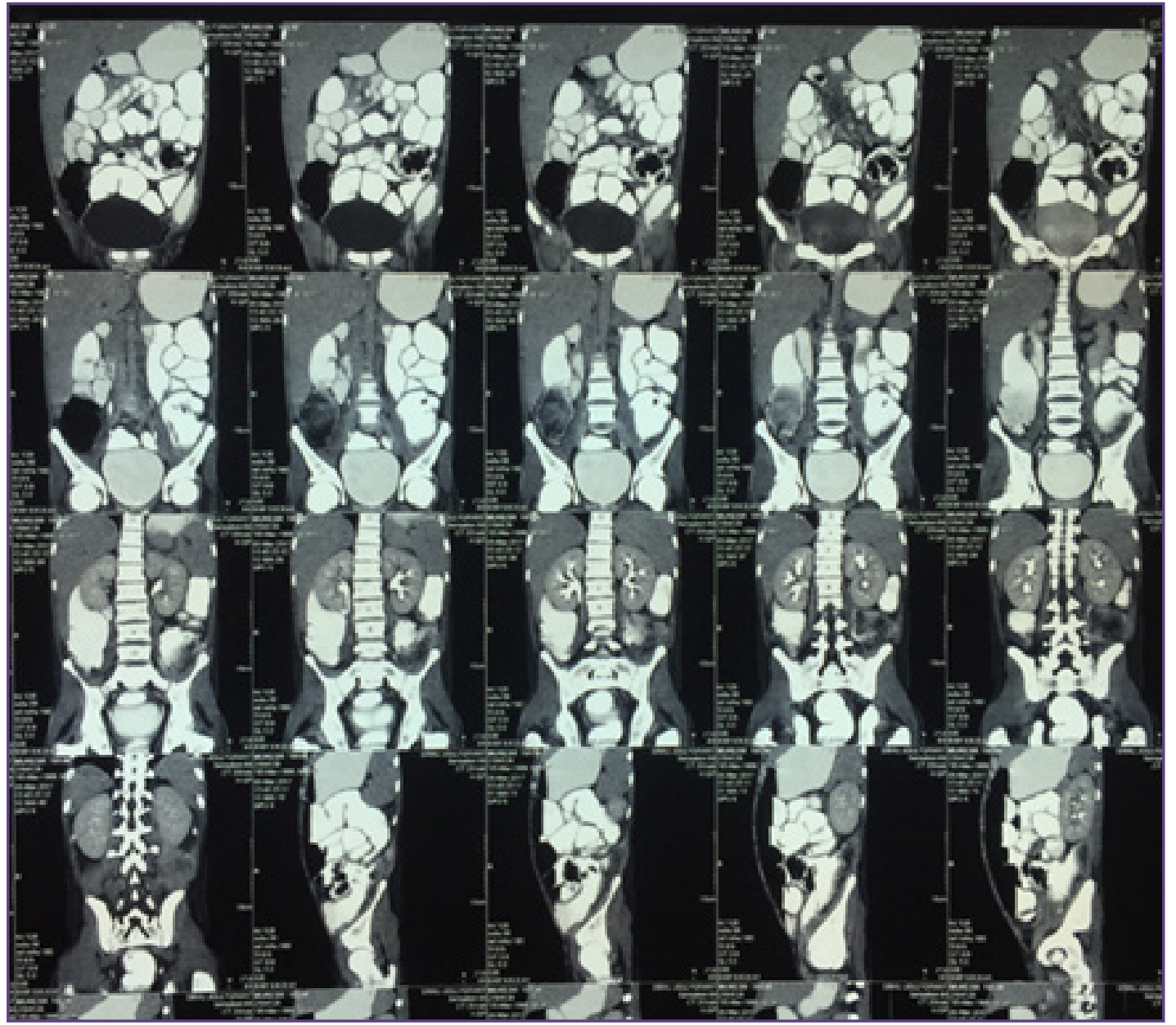

Fig. 1: CECT abdomen showing hepatomegaly, retroperitoneal lymphadenopathy and collection of faecal matter in the peritoneum.

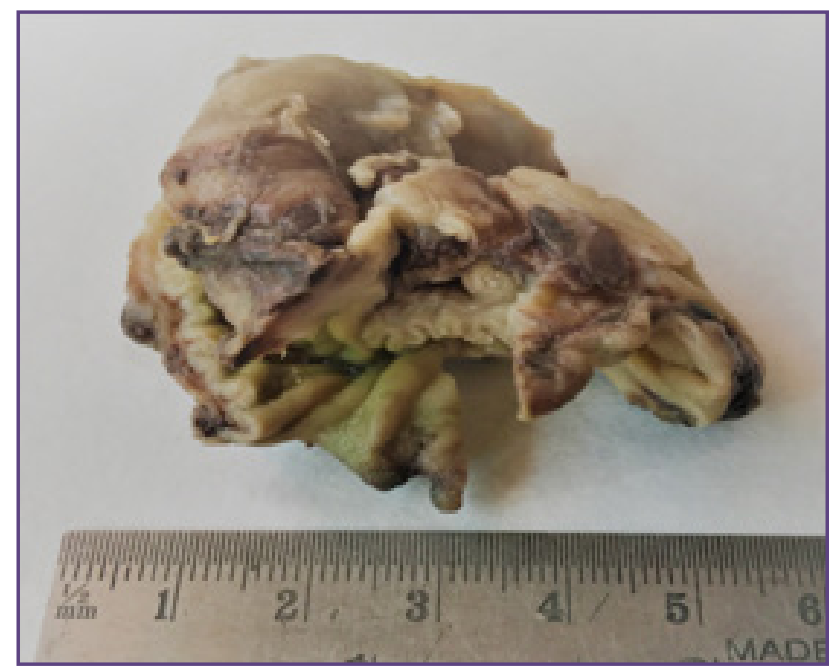

Fig. 2:Segment of ileum with an area of perforation.

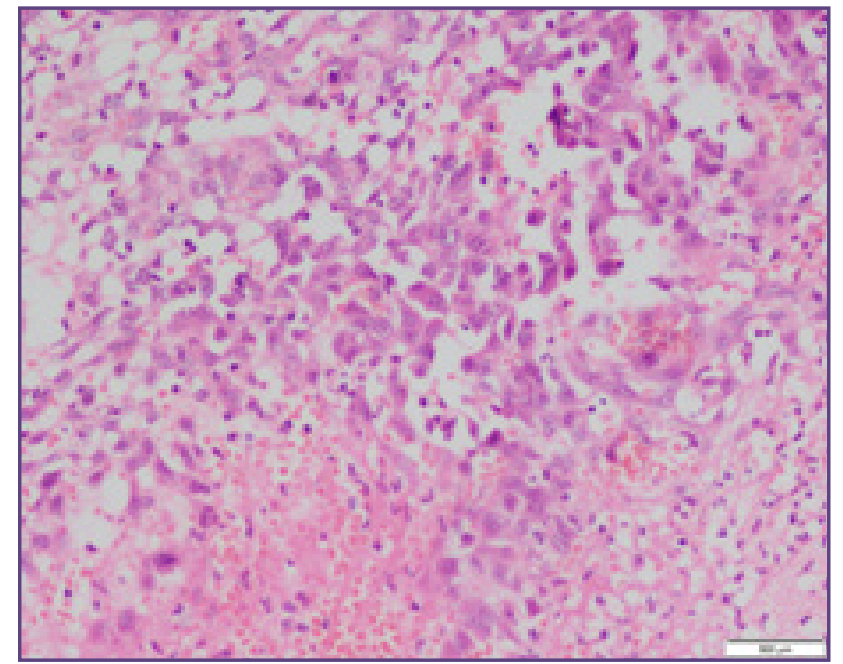

Fig. 3: Photomicrograph showing atypical cells in submucosa (myeloblasts) exhibiting high $\mathrm{N}: \mathrm{C}$ ratio and few prominent nucleoli. H\&E.4x10X. 


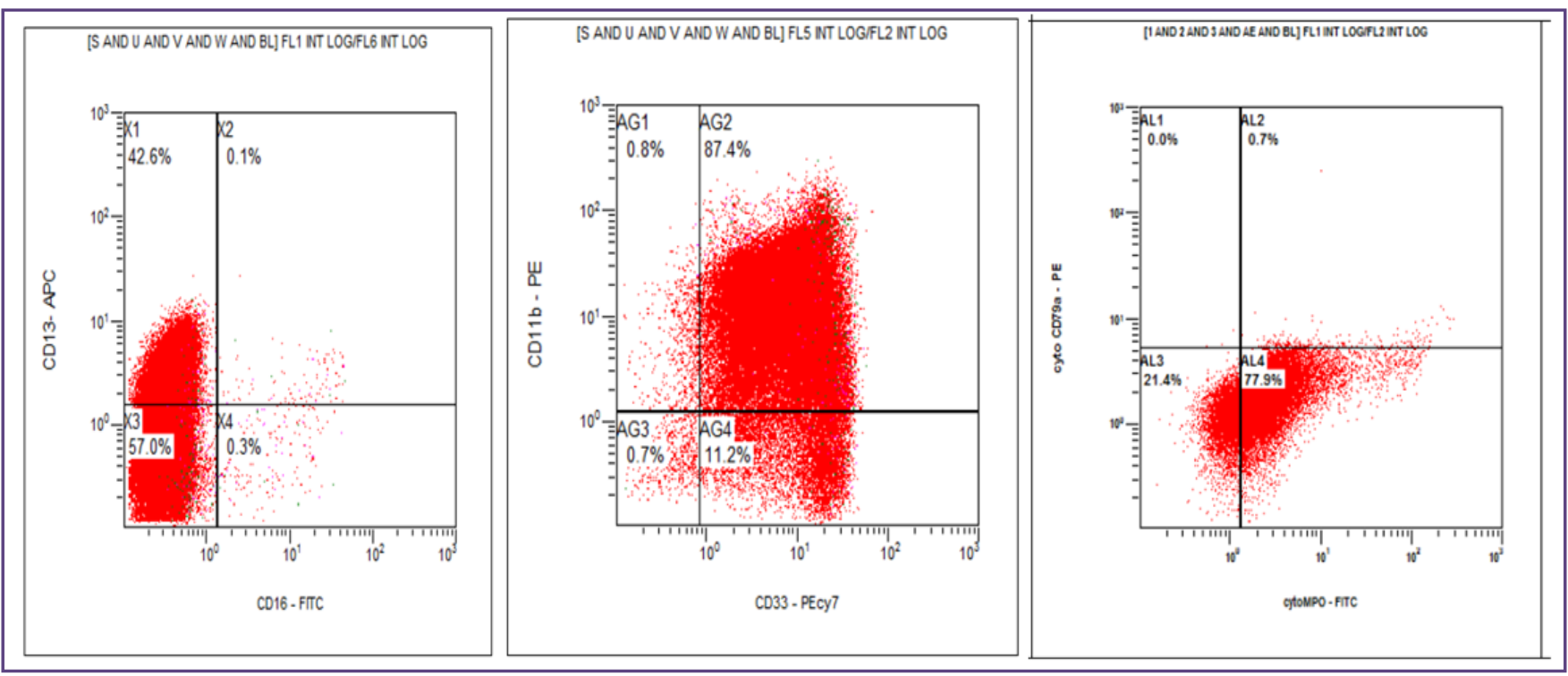

Fig. 4: Flow cytometry exhibiting cytoplasmic MPO,CD 13 and CD33 positivity.

form of treated AML. It may also signal impending blast crisis in the setting of a myeloproliferative disorder or leukemic transformation in myelodysplastic syndrome. ${ }^{[3,4]}$ Less commonly, it may also occur as an isolated mass in nonleukemic patients (primary GS). The average period of transformation to AML in non leukemic patients is 11 months. ${ }^{[5]}$ It is more frequently associated with acute myeloblastic leukemia with maturation (FrenchAmericanBritish [FAB] M2) but can also be seen in other subtypes many previous studies have described GS of small intestine with an average age of presentation ranging from 8 to 69 years (mean, 43 years), with the majority occurring in male patients (17/ 20 cases). ${ }^{[4]}$ Ileal involvement has been described in $65 \%$ of the cases however upto 4 organs at a time have been reported. ${ }^{[5,6]}$ One mass developed in a 52 -year-old man who had been in complete remission for AML M2 for more than 5 years. ${ }^{[6]}$ It is more common in children and young adults as was seen in a 17 year old boy in Lahore, Pakistan. ${ }^{[7]}$ In a Korean series of 41 patients with MS, the majority of patients were reported to be younger than 40 years. ${ }^{[8]}$ Myeloid sarcoma of the gastrointestinal tract may macroscopically appear in the form of wall thickening, polypoid mass, exophytic mass or ulcerated lesions. The most common site of involvement in the gastrointestinal tract is the small bowel. The World Health Organization classifies MS that is a hematopoietic tumor under two main headings: GS is the more common form that is composed of myeloblasts, neutrophils and myeloid precursors, whereas monoblastic sarcoma is a rare form that is composed of monoblasts and is associated with acute monoblastic sarcoma. ${ }^{[3]}$ Isolated MS can be mistaken for a variety of lymphoma and hematopoietic tumors, however, with the introduction of specific immunohistochemical markers such as myeloperoxidase, chloroacetate esterase, lysozyme, CD43, CD13, CD33, and CD117 diagnostic difficulties and errors are greatly diminished in recent years . ${ }^{[9]}$ The treatment options as well as prognosis of myeloid sarcoma of gastrointestinal involvement remain the same as that of AML that is, systemic chemotherapy with bone marrow transplantation in addition to surgical resection in appropriate clinical settings. Systemic chemotherapy provides the most benefit. ${ }^{[10]}$

\section{Conclusion}

Hematogenous malignancy particularly AML can present with extramedullary deposits of myeloblasts. Such cases may also precede the development of leukemia and hence require high degree of clinical suspicion and warrant a complete laboratory work up.

\section{References}

1. Burns A: Observations of surgical anatomy, head and neck. In: Royce T (ed). Edinburgh, United Kingdom, Thomas Royce and Company 1811; pp 364-6.

2. Davey FR, Olson S, Kurec AS, Eastman-Abaya R, Gottlieb AJ, Mason DY. The immunophenotyping of extramedullary myeloid cell tumors in paraffin embedded tissue sections. Am T Surg Pathol 1988; 12: 699-707.

3. Brunning RD, Matutes E, Flandrin G, et al. Acute myeloid leukaemia not otherwise categorised. In: Jaffe ES, Harris NL, Stein H, Vardiman JW, eds. Tumours of Haematopoietic Tissues.Lyon, France: IARC Press; 2001:104-105. World Health Organization Classification of Tumours.

4. Guermazi A, Feger C, Rousselot P. Granulocytic Sarcoma (Chloroma): Imaging Findings in Adults and Children. Am J Roentgenol 2002; 178: 319-25. 
5. Yamauchi K, Yasuda M. Comparison in treatments of nonleukemic granulocytic sarcoma: report of two cases and a review of 72 cases in the literature. Cancer. 2002;94:1739-46.

6. Kohl SK, Aoun P. Granulocytic Sarcoma of the Small Intestine. Arch Path Lab Med 2006; 130: 1570-4.

7. Tariq G, Alia Z, Ibrahim Al N. Granulocytic sarcoma of small intestine:An unusual presentation of Acute Myelogenous Leukaemia. J Pak Med Assoc 2010;60(2) 133-36
8. Jung SH, Kim HC, Yu CS, Kim JC. Solitary preleukemic granulocytic sarcoma as a cause of small bowel obstruction. Gut Liver. 2007;1:82-86.

9. Erkut N, Vetem İ, Çobanoğlu Ü, Sönmez M. Presentation of acutemyeloid myeloid leukemia preceded by myelodysplastic syndrome with multiple extramedullary infiltration: Case report. Acta Oncologica Turcica. 2009;42:121-124.

10. Martinelli G, Vianelli N, De Vivo A, et al. Granulocytic sarcomas: clinical, diagnostic, and therapeutical aspects. Leuk Lymphoma. 1997;24:349-53.

*Corresponding author:

Dr. Nadia Shirazi, Professor, Department of Pathology, Himalayan Institute of Medical Sciences, Jolly Grant, Dehradun. INDIA, Pin: 248140

Phone: +91 9758376477

Email: shirazinadia@gmail.com

Financial or other Competing Interests: None. 